\title{
Presurgical Nasoalveolar Moulding-An Adjunct to Facilitate Surgical Repair in Infants with Cleft Lip and Palate
}

\author{
Dolly Patel, Ramesh Goyal, Taruna Puri
}

Department of Orthodontics and Dentofacial Orthopaedics, Government Dental College and Hospital, Ahmedabad, India.

Email: tarunapuri5@gmail.com,dollypankaj@gmail.com

Received October $11^{\text {th }}, 2012$; revised November $13^{\text {th }}, 2012$; accepted December $15^{\text {th }}, 2012$

\begin{abstract}
Presurgical orthopedics forms an integral part of the treatment of patients with facial clefting. The primary aim of presurgical nasoalveolar moulding (PNAM) is reduction in the soft tissue and cartilaginous deformity to facilitate surgical soft tissue repair in optimal conditions under minimum tension to minimize scar formation. It also helps in reducing the nasal deformity with a nasal stent which brings the deformity near to the normal and forms an important part of the primary nasal repair at the time of primary lip repair. A soft acrylic relining material is added or removed to line the appliance to a thickness of approximately $1-1.5 \mathrm{~mm}$ in the region from where the bone is to be resorbed or apposed. This article describes the fabrication procedure of the modified nasal alveolar molding appliance in eight infants with unilateral cleft lip and palate in whom a significant reduction in cleft width and nasoalveolar deformity was achieved. The results showed that the greater segment shows more favourable changes and can be molded more positively and efficiently than the lesser segment.
\end{abstract}

Keywords: PNAM; Nasal Stent; Pre-Surgical Orthopaedics

\section{Introduction}

Cleft lip and palate is the most common congenital craniofacial anomaly caused by abnormal facial development during gestation. Cleft lip and palate though treatable; it has a great negative social impact on the patient as well as his/her family. However, the kind of treatment of cleft lip and palate depends on the type of cleft and the severity of the cleft.

The global epidemiological survey states that cleft palate is present in one in every 600 newborns. United States Bureau of the Census (2001) says that a child with cleft is born every $2.5 \mathrm{~min}$. Incidence is highest amongst the Asians followed by Caucasians and Africans. In India, over $3500 \mathrm{CLP} / \mathrm{yr}$ are reported.

Most children with a form of clefting are monitored by a cleft palate team or craniofacial team from birth to young adulthood. The overall care of affected infant should rely on interdisciplinary team decisions rather than a series of independent, critical events by individual specialists on a team. Cleft palate team or craniofacial team consists of a plastic surgeon, an orthodontist, a speech therapist, a paediatrician, oral and maxillofacial surgeon, a prosthodontist, nursing staff and a team coordinator. Plastic surgeon, orthodontist, and speech thera- pist constitute part of the core team. The orthodontist forms an integral part of the habilitative process. The role of an orthodontist is to monitor the craniofacial growth \& development, to correct jaw relationship \& dental occlusion and finally to achieve optimal functional occlusion, appearance \& stability.

Presurgical orthodontics or neonatal maxillary orthopedics is initiated during the first or second week following birth. The perspective of contemporary wisdom is that when provided as an adjunctive procedure to primary definitive lip repair, neonatal maxillary orthopedics does have presurgical benefits.

The primary aim of presurgical nasoalveolar moulding (PNAM) is reduction in the soft tissue and cartilaginous deformity to facilitate surgical soft tissue repair in optimal conditions under minimum tension to minimize scar formation [1,2]. It allows stimulation and redirection of growth for the controlled predictable repositioning of the alveolar segments and gives the ideal arch form, normalizes the tongue position, aids in speech development, improves appearance and gives a psychosocial boost, improves feeding and bone contour.

From the frontal view the faces are found to be asymmetric due to unilateral complete cleft involving lip and 
alveolus, there is a deviation of nasal tip from the facial midline and a deformed nasal dome with significant flattening, columellar shortening with deviation and philtral shortening. The lower lateral alar cartilage on the cleft side is often concave where it ought to be convex. The nostril apex is low on the cleft side, and the alar rim is stretched in the horizontal plane. Intraoral view revealed extension of the cleft till the posterior extent of the hard palate and involving the soft palate too.

\subsection{Problems on the Cleft Side}

$>$ Premaxilla outwardly rotated/projected.

$>$ Lateral segment placed posteriorly.

$>$ Inferior septum out of vomer groove-spine in floor of normal nostril.

$>$ Unilateral shortness of height columella.

$>$ Short philtrum.

$>$ Orbicularis oris is disrupted and ends in margin of cleft.

\subsection{Problems on the Non-Cleft Side}

Shortened philtral height.

$>$ Short columella.

> 2/3 of cupids bow, 1 philtral column preserved.

$>$ Muscle between midline and cleft side (Figure 1).

Correction of the cleft nasal deformity has been the greatest challenge to the cleft surgeons. Nasal deformity increases with the time if left untreated [3].

Unrepaired nasal deformity also leaves the stigmata of cleft till the adolescence. Also secondary correction of the nasal deformity would lead to more surgical scarring and less than ideal results [4].Thus, any form of the nonsurgical treatment to reduce the nasal deformity early in the life is highly desirable. First attempt at the presurgical nasal correction was made by Matsuo et al. $[5,6]$.

Model analysis revealed a disrupted arch form with lesser segment being placed at the posterior end and a greater segment oriented outward and laterally (Figure 2).

\section{Treatment Objectives}

The primary objective of the treatment is stimulation and redirection of alveolar growth aiming for the non surgical approximation of the cleft deformity. To achieve this it is planned to treat the patients with moulding appliance in conjunction with a traction force across the cleft lip followed by a nasal stent later.The nasal stent and alveolar moulding plate help in achieving nasal and alveolar symmetry, nasal tip projection, and contact of the cleft alveolus just before primary lip, nasal and alveolar surgical repair.
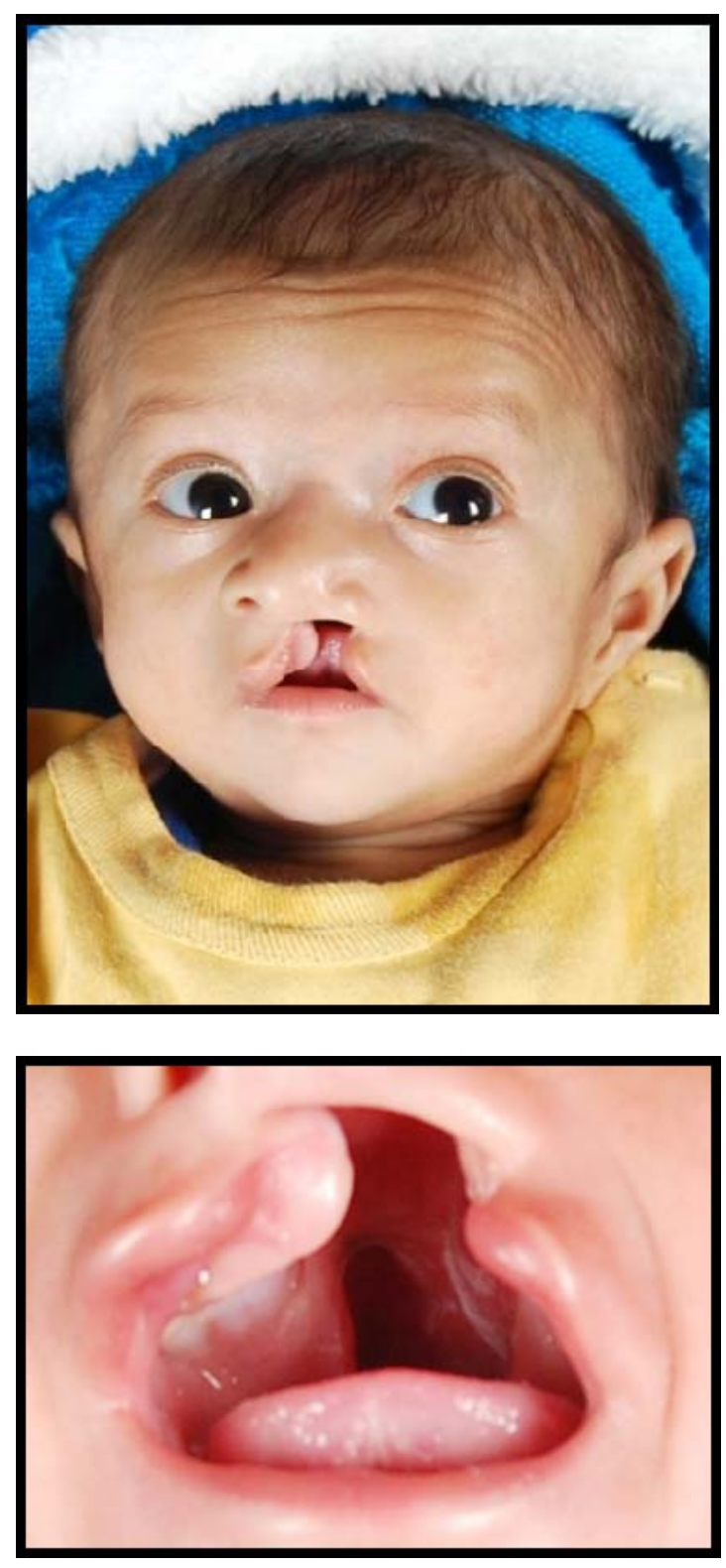

Figure 1. Frontal extraoral view and intraoral view of a unilateral complete left side cleft in an infant.

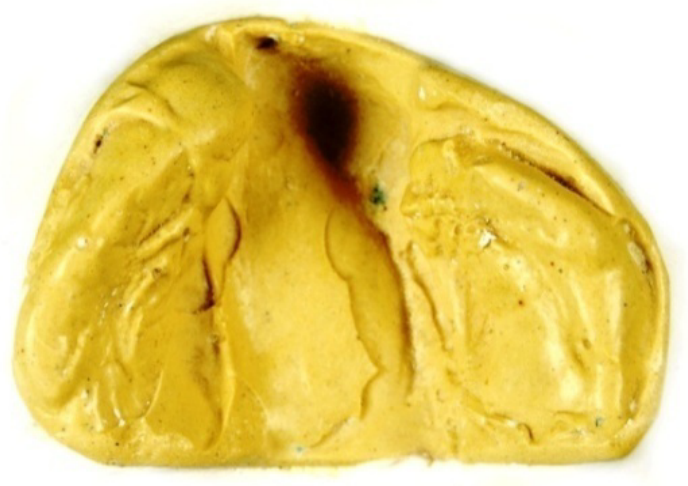

Figure 2. Figure showing the disrupted arch form. 


\section{Materials and Methods}

This article describes infants with unilateral cleft lip, alveolus and palate in whom presurgical nasoalveolar moulding was done.

The primary impression is taken with impression compound followed by the final impression on the same day with elastomeric impression material using a custom tray. Patient positioning is an important part of the overall procedure. The infant is held upside down in the mother's lap in such a way that the infant's neck can be extended for the maximum exposure for the operator. Care should be taken to support the infant's head. This positioning helps to visualize the posterior extent of the tray. Flow of the impression material should be controlled so that it does not go beyond the tray boundaries. Through-out the procedure it is made sure that the infant is actively crying. It helps to avoid the choking of infant's airway and also makes direct visualization of the cleft possible [7]. The impression is poured with dental stone and is duplicated to obtain two sets of models, one to serve as a record model and other for making the moulding plate. A molding plate, made up of hard clear acrylic is fabricated on the dental stone model. This molding plate differs from the conventional orthopedic plate as described by the Hotz [8]. Molding plate is fabricated in such a way that there are no extensions of the plate into the alveolar or palatal cleft space. All the undercuts and the cleft space is obliterated with the modelling wax so that cast appears to have an intact alveolus. At the same time two to three layers of the modelling wax is also added as a spacer in the region where the major segments has to move on the palatal side during the course of the treatment. Obliteration of the cleft space and the addition of wax spacer is the key modification.

The feeding plate is delivered on day 2 for permanent wear except for cleaning. It is relieved in the region of overextensions. It is maintained in the position in the mouth of infants $24 \mathrm{hrs}$ a day and removed only for cleaning after feeds. On this visit the parents are instructed to start tapping the lip across the cleft gap, between the maxillary lip segments with force vector directed towards the posterior end and superiorly (Figure 3). While a lip adhesion alone produces uncontrolled orthopaedic effects, a lip taping force, in conjunction with a moulding plate, yields a controlled movement of alveolar segments in a predetermined direction.

The first adjustment is scheduled on the day of plate delivery. The tissue surface of the appliance is modified to begin the moulding of the greater and lesser alveolar segments on either side of the cleft by selective removal of acrylic from the region where bony apposition is desired. At the same time, a soft acrylic relining material is added to line the appliance to a thickness of approxi-
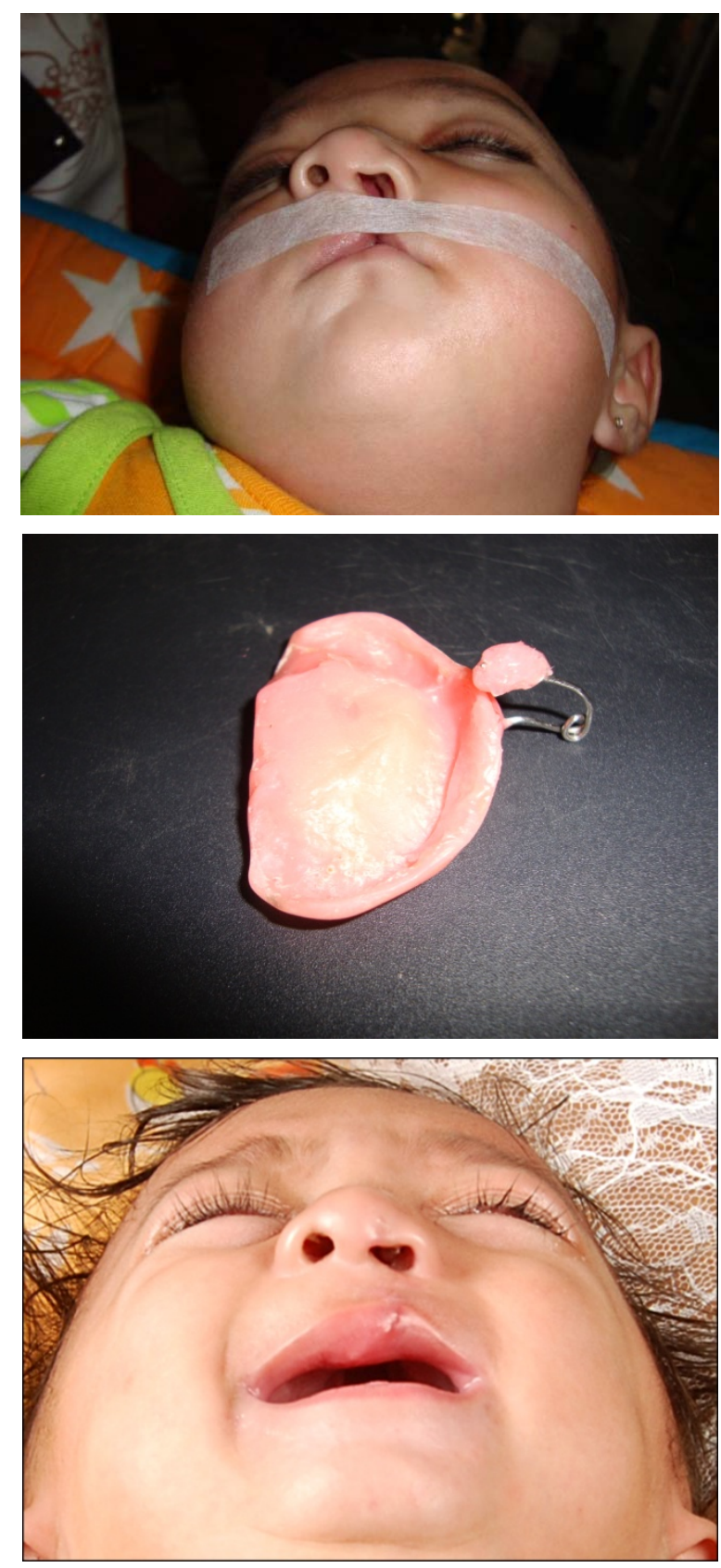

Figure 3. Maxillary plate with attached nasoalveolar stent and primary lip tape in position.

mately $1-1.5 \mathrm{~mm}$ in the region from where we want the bone to be resorbed. The recommended action is to direct the greater segment inward towards the cleft and the lesser segment outward from the cleft. These minor adjustments are made on a weekly basis after careful consideration of the cleft situation (Figure 4).

After 7 weeks of active nasoalveolar moulding, an alveolar cleft width reduction is achieved, after which the nasal stent is added to the labial flange of the moulding plate for nasal cartilage moulding into a swan neck configuration and embedded into the plate at the cleft site, 


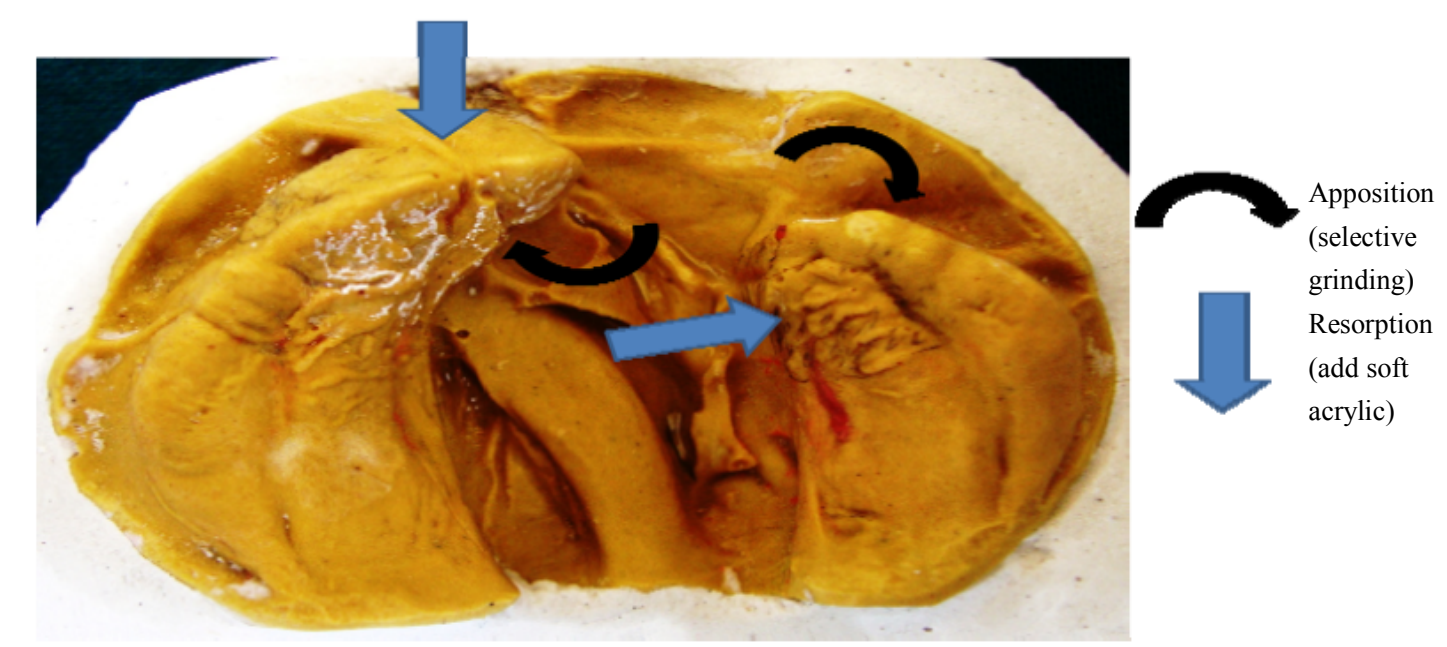

Figure 4. Figure showing apposition and resorption.

containing a loop for activation, which lift the nasal dome near the nasal tip (Figure 5).

Aggressive lip taping is continued even after the nasal stent had been added to the appliance. Moulding treatment using the plate and lip taping was continued until the time of surgical lip repair.

The appliance is worn continuously except for cleansing after feeding. The retention tapes and lip tapes are changed as soon as they become loose.

Through this procedure, the shape of the cartilaginous septum, alar tip and medial and lateral crus are carefully moulded to resemble the normal shape of these structures. The impressions are taken prior to surgery and the plate is discontinued after surgery

Model analysis was done by marking points GA, LA, GM, LM, GP and LP on the models and taking the linear distances between them as shown in Figure 6. Measurements of these records were done 3 times during the first 5 months of treatment, which revealed a gradual cleft reduction in alveolus and in the palatal segments.

GA: Most anterior point on the greater segment.

GP: Most posterior point on the greater segment.

LA: Most anterior point on the lesser segment.

LP: Most posterior point on the lesser segment.

GA-LA: Alveolar cleft gap.

GP-LP: Posterior transverse alveolar width.

MG: Deepest point on medial border of greater segment.

ML: Deepest point on medial outline of lesser segment.

The linear distance between GA and LA point which shows the anterior cleft width was measured. The mean reduction in GA-LA from pretreatment to presurgical model was $3.17 \mathrm{~mm}$ and from pretreatment to post surgical model was $6.75 \mathrm{~mm}$ (Table 1).

The linear distance between GM and LM point which shows the cleft width at middle portion of the alveolus
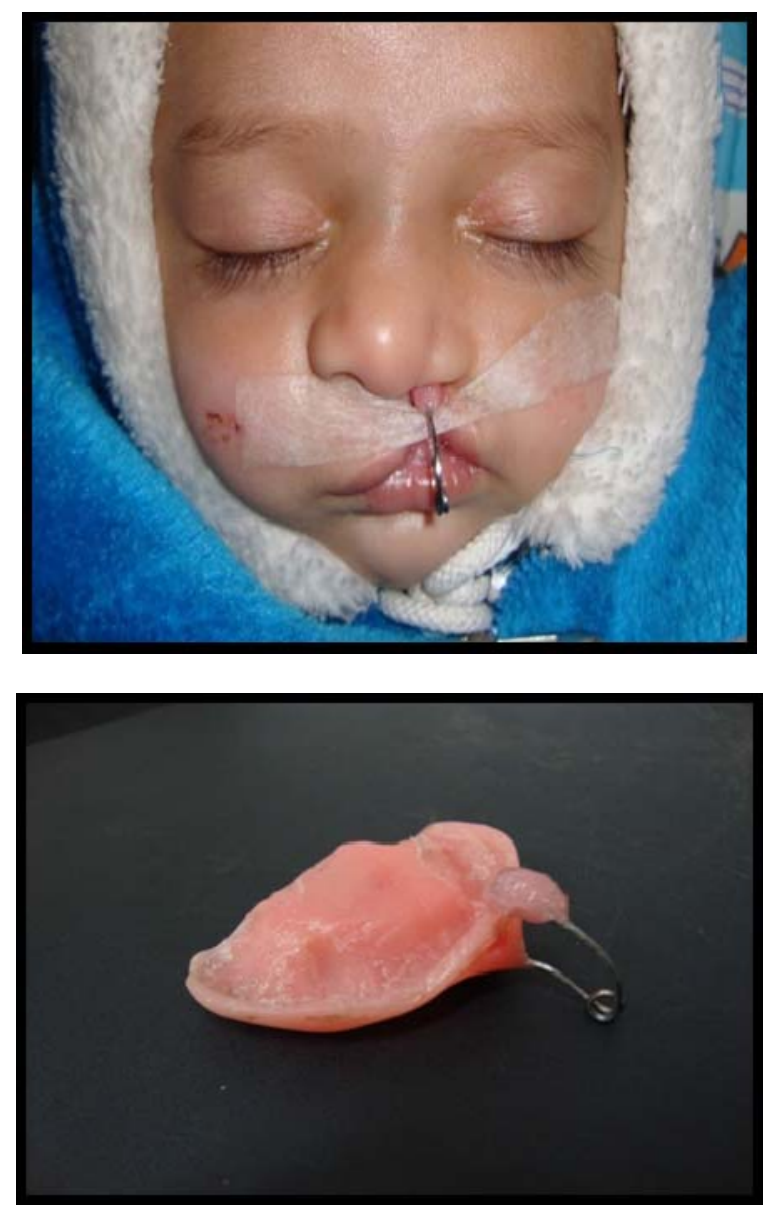

Figure 5. Nasal stent.

was measured. The mean reduction in GM-LM from pretreatment to presurgical model was $1.83 \mathrm{~mm}$ and from pretreatment to post surgical model was $4.17 \mathrm{~mm}$ (Table 1).

The linear distance between GP and LP point which 

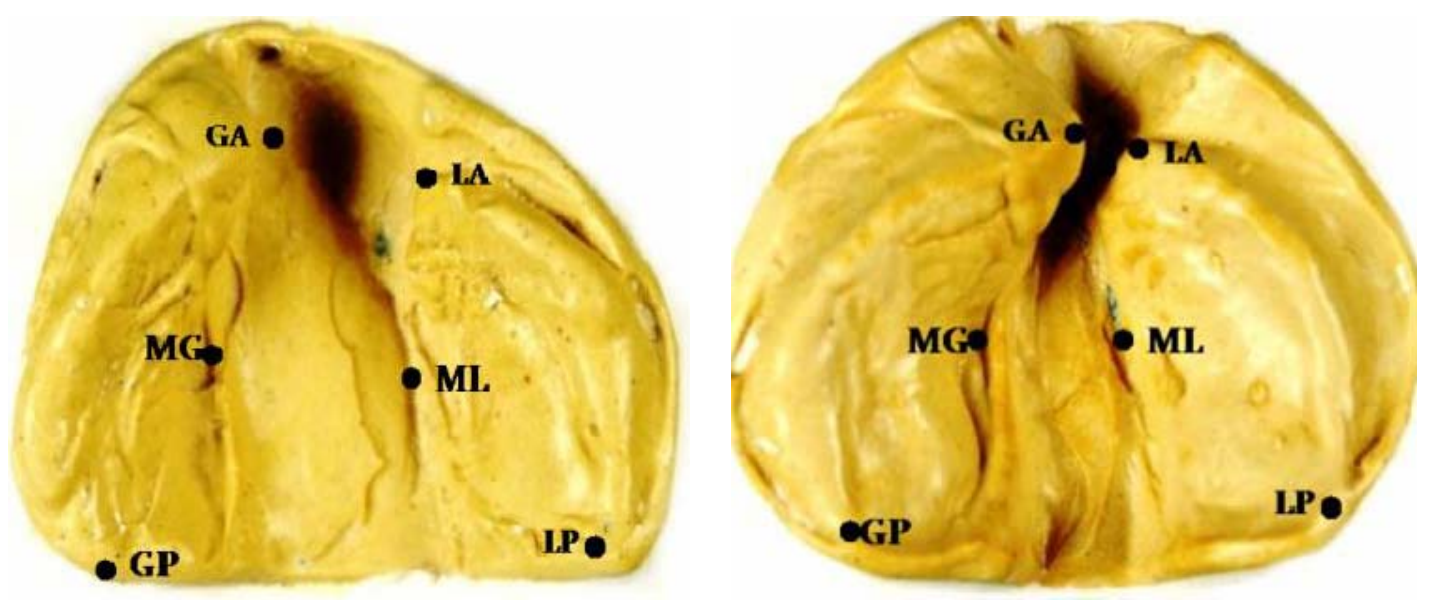

Figure 6. Figure showing the points used for model analyses.

Table 1. Paired samples test.

\begin{tabular}{|c|c|c|c|c|c|c|c|c|c|c|}
\hline & \multicolumn{5}{|c|}{ Paired Differences } & \multirow{3}{*}{$\mathrm{T}$} & \multirow{3}{*}{ Df } & \multirow{3}{*}{$P$ value } & \\
\hline & & \multirow[t]{2}{*}{ Mean } & \multirow{2}{*}{$\begin{array}{c}\text { Std. } \\
\text { Deviation }\end{array}$} & \multirow{2}{*}{$\begin{array}{l}\text { Std. Error } \\
\text { Mean }\end{array}$} & \multicolumn{2}{|c|}{$\begin{array}{l}95 \% \text { Confidence } \\
\text { Interval of the } \\
\text { Difference }\end{array}$} & & & & \\
\hline & & & & & Lower & Upper & & & & \\
\hline Pair 1 & GA_LA_PRERX-GA_LA_PRESURG & 3.000 & 1.690 & 0.598 & 1.587 & 4.413 & 5.020 & 7 & 0.002 & Significant \\
\hline Pair 2 & GA_LA_PRERX-GA_LA_POSTSURG & 6.750 & 2.485 & 1.014 & 4.142 & 9.358 & 6.654 & 5 & 0.001 & Significant \\
\hline Pair 3 & GA_LA_PRESURG-GA_LA_POSTSURG & 3.750 & 2.139 & 0.873 & 1.505 & 5.995 & 4.294 & 5 & 0.008 & Significant \\
\hline Pair 4 & GM_LM_PRERX-GM_LM_PRESURG & 2.375 & 2.446 & 0.865 & 0.330 & 4.420 & 2.747 & 7 & 0.029 & Significant \\
\hline Pair 5 & GM_LM_PRERX-GM_LM_POSTSURG & 4.167 & 1.722 & 0.703 & 2.359 & 5.974 & 5.926 & 5 & 0.002 & Significant \\
\hline Pair 6 & GM_LM_PRESURG-GM_LM_POSTSURG & 2.333 & 1.751 & 0.715 & 0.496 & 4.171 & 3.264 & 5 & 0.022 & Significant \\
\hline Pair 7 & GP_LP_PRERX-GP_LP_PRESURG & -1.125 & 1.885 & 0.666 & -2.701 & 0.451 & -1.688 & 7 & 0.135 & Non-significant \\
\hline Pair 8 & GP_LP_PRERX-GP_LP_POSTSURG & -0.500 & 1.975 & 0.806 & -2.572 & 1.572 & -0.620 & 5 & 0.562 & Non-significant \\
\hline Pair 9 & GP_LP_PRESURG-GP_LP_POSTSURG & -0.333 & 1.966 & 0.803 & -2.397 & 1.730 & -0.415 & 5 & 0.695 & Non-significant \\
\hline Pair 10 & GA_GP_PRERX-GA_GP_PRESURG & -3.250 & 3.284 & 1.161 & -5.996 & -0.504 & -2.799 & 7 & 0.027 & Significant \\
\hline Pair 11 & GA_GP_PRERX-GA_GP_POSTSURG & -6.000 & 4.648 & 1.897 & -10.87 & -1.123 & -3.162 & 5 & 0.025 & Significant \\
\hline Pair 12 & GA_GP_PRESURG-GA_GP_POSTSURG & -2.667 & 3.882 & 1.585 & -6.740 & 1.407 & -1.683 & 5 & 0.153 & Non-significant \\
\hline Pair 13 & LA_LP_PRERX-LA_LP_PRESURG & -1.875 & 3.182 & 1.125 & -4.535 & 0.785 & -1.667 & 7 & 0.140 & Non-significant \\
\hline Pair 14 & LA_LP_PRERX-LA_LP_POSTSURG & -4.333 & 2.338 & 0.955 & -6.787 & -1.880 & -4.540 & 5 & 0.006 & Significant \\
\hline Pair 15 & LA_LP_PRESURG-LA_LP_POSTSURG & -3.000 & 2.530 & 1.033 & -5.655 & -0.345 & -2.905 & 5 & 0.034 & Significant \\
\hline
\end{tabular}

shows the posterior transverse alveolar width was measured. The mean increase in GP-LP from pretreatment to presurgical model was $0.17 \mathrm{~mm}$ and from pretreatment to post surgical model was $0.5 \mathrm{~mm}$ (Table 1).

The linear distance between GA and GP point which shows the length of the greater segment was measured. The mean increase in GA-GP from pretreatment to presurgical model was $3.33 \mathrm{~mm}$ and from pretreatment to post surgical model was $6 \mathrm{~mm}$ (Table 1).
The linear distance between LA and LP point which shows the length of the lesser segment was measured. The mean increase in LA-LP from pretreatment to presurgical model was $1.33 \mathrm{~mm}$ and from pretreatment to post surgical model was $4.33 \mathrm{~mm}$ (Table 1).

The statistical analysis used in the study was the paired t-test. The differences between the pretreatment, presurgical and post surgical measurements of the cleft defect were calculated, their mean, standard deviation and 
standard error of mean were calculated and $\mathrm{p}$ value was calculated (Tables 1-3).

\section{Results}

The greater alveolar segment shows sagittal growth towards the midsagittal plane more than the lesser segment.

Also from the above results it is evident that the greater segment shows more favourable changes and can be molded more positively and efficiently than the lesser segment (Figure 7).

Photographic analysis reveals an improvement in the vertical level of nasal alae. Discrepancy in the vertical level of both sides of alae reduced before surgery. Nasal tip deviation from the midsagittal reference plane reduced prior to surgery and became nil after surgery. The ala of the nose showed an improvement of ensuring symmetrical nasal morphology (Figure 8).

Table 2. Paired samples statistics.

\begin{tabular}{|c|c|c|c|c|c|}
\hline & & Mean & $\mathrm{N}$ & Std. Deviation & Std. Error Mean \\
\hline \multirow{2}{*}{ Pair 1} & GA_LA_PRERX & 9.00 & 8 & 3.381 & 1.195 \\
\hline & GA_LA_PRESURG & 6.00 & 8 & 2.449 & 0.866 \\
\hline \multirow{2}{*}{ Pair 2} & GA_LA_PRERX & 9.17 & 6 & 3.312 & 1.352 \\
\hline & GA_LA_POSTSURG & 2.42 & 6 & 1.357 & 0.554 \\
\hline \multirow{2}{*}{ Pair 3} & GA_LA_PRESURG & 6.17 & 6 & 2.401 & 0.980 \\
\hline & GA_LA_POSTSURG & 2.42 & 6 & 1.357 & 0.554 \\
\hline \multirow{2}{*}{ Pair 4} & GM_LM_PRERX & 14.63 & 8 & 2.134 & 0.754 \\
\hline & GM_LM_PRESURG & 12.25 & 8 & 1.282 & 0.453 \\
\hline \multirow{2}{*}{ Pair 5} & GM_LM_PRERX & 14.50 & 6 & 2.168 & 0.885 \\
\hline & GM_LM_POSTSURG & 10.33 & 6 & 2.251 & 0.919 \\
\hline \multirow{2}{*}{ Pair 6} & GM_LM_PRESURG & 12.67 & 6 & 1.033 & 0.422 \\
\hline & GM_LM_POSTSURG & 10.33 & 6 & 2.251 & 0.919 \\
\hline \multirow{2}{*}{ Pair 7} & GP_LP_PRERX & 33.88 & 8 & 3.980 & 1.407 \\
\hline & GP_LP_PRESURG & 35.00 & 8 & 4.276 & 1.512 \\
\hline \multirow{2}{*}{ Pair 8} & GP_LP_PRERX & 34.00 & 6 & 4.690 & 1.915 \\
\hline & GP_LP_POSTSURG & 34.50 & 6 & 3.017 & 1.232 \\
\hline \multirow{2}{*}{ Pair 9} & GP_LP_PRESURG & 34.17 & 6 & 4.622 & 1.887 \\
\hline & GP_LP_POSTSURG & 34.50 & 6 & 3.017 & 1.232 \\
\hline \multirow{2}{*}{ Pair 10} & GA_GP_PRERX & 32.50 & 8 & 3.928 & 1.389 \\
\hline & GA_GP_PRESURG & 35.75 & 8 & 3.615 & 1.278 \\
\hline \multirow{2}{*}{ Pair 11} & GA_GP_PRERX & 33.00 & 6 & 4.472 & 1.826 \\
\hline & GA_GP_POSTSURG & 39.00 & 6 & 5.020 & 2.049 \\
\hline \multirow{2}{*}{ Pair 12} & GA_GP_PRESURG & 36.33 & 6 & 4.033 & 1.647 \\
\hline & GA_GP_POSTSURG & 39.00 & 6 & 5.020 & 2.049 \\
\hline \multirow{2}{*}{ Pair 13} & LA_LP_PRERX & 23.38 & 8 & 2.774 & 0.981 \\
\hline & LA_LP_PRESURG & 25.25 & 8 & 4.334 & 1.532 \\
\hline \multirow{2}{*}{ Pair 14} & LA_LP_PRERX & 24.50 & 6 & 2.168 & 0.885 \\
\hline & LA_LP_POSTSURG & 28.83 & 6 & 3.061 & 1.249 \\
\hline \multirow{2}{*}{ Pair 15} & LA_LP_PRESURG & 25.83 & 6 & 4.446 & 1.815 \\
\hline & LA_LP_POSTSURG & 28.83 & 6 & 3.061 & 1.249 \\
\hline
\end{tabular}


Table 3. Paired samples correlations.

\begin{tabular}{|c|c|c|c|c|}
\hline & & $\mathrm{N}$ & Correlation & Sig. \\
\hline Pair 1 & GA_LA_PRERX \& GA_LA_PRESURG & 8 & 0.880 & 0.004 \\
\hline Pair 2 & GA_LA_PRERX \& GA_LA_POSTSURG & 6 & 0.738 & 0.094 \\
\hline Pair 3 & GA_LA_PRESURG \& GA_LA_POSTSURG & 6 & 0.465 & 0.352 \\
\hline Pair 4 & GM_LM_PRERX \& GM_LM_PRESURG & 8 & 0.039 & 0.927 \\
\hline Pair 5 & GM_LM_PRERX \& GM_LM_POSTSURG & 6 & 0.697 & 0.124 \\
\hline Pair 6 & GM_LM_PRESURG \& GM_LM_POSTSURG & 6 & 0.660 & 0.154 \\
\hline Pair 7 & GP_LP_PRERX \& GP_LP_PRESURG & 8 & 0.898 & 0.002 \\
\hline Pair 8 & GP_LP_PRERX \& GP_LP_POSTSURG & 6 & 0.961 & 0.002 \\
\hline Pair 9 & GP_LP_PRESURG \& GP_LP_POSTSURG & 6 & 0.954 & 0.003 \\
\hline Pair 10 & GA_GP_PRERX \& GA_GP_PRESURG & 8 & 0.624 & 0.098 \\
\hline Pair 11 & GA_GP_PRERX \& GA_GP_POSTSURG & 6 & 0.526 & 0.284 \\
\hline Pair 12 & GA_GP_PRESURG \& GA_GP_POSTSURG & 6 & 0.652 & 0.161 \\
\hline Pair 13 & LA_LP_PRERX \& LA_LP_PRESURG & 8 & 0.680 & 0.063 \\
\hline Pair 14 & LA_LP_PRERX \& LA_LP_POSTSURG & 6 & 0.648 & 0.164 \\
\hline Pair 15 & LA_LP_PRESURG \& LA_LP_POSTSURG & 6 & 0.835 & 0.038 \\
\hline
\end{tabular}
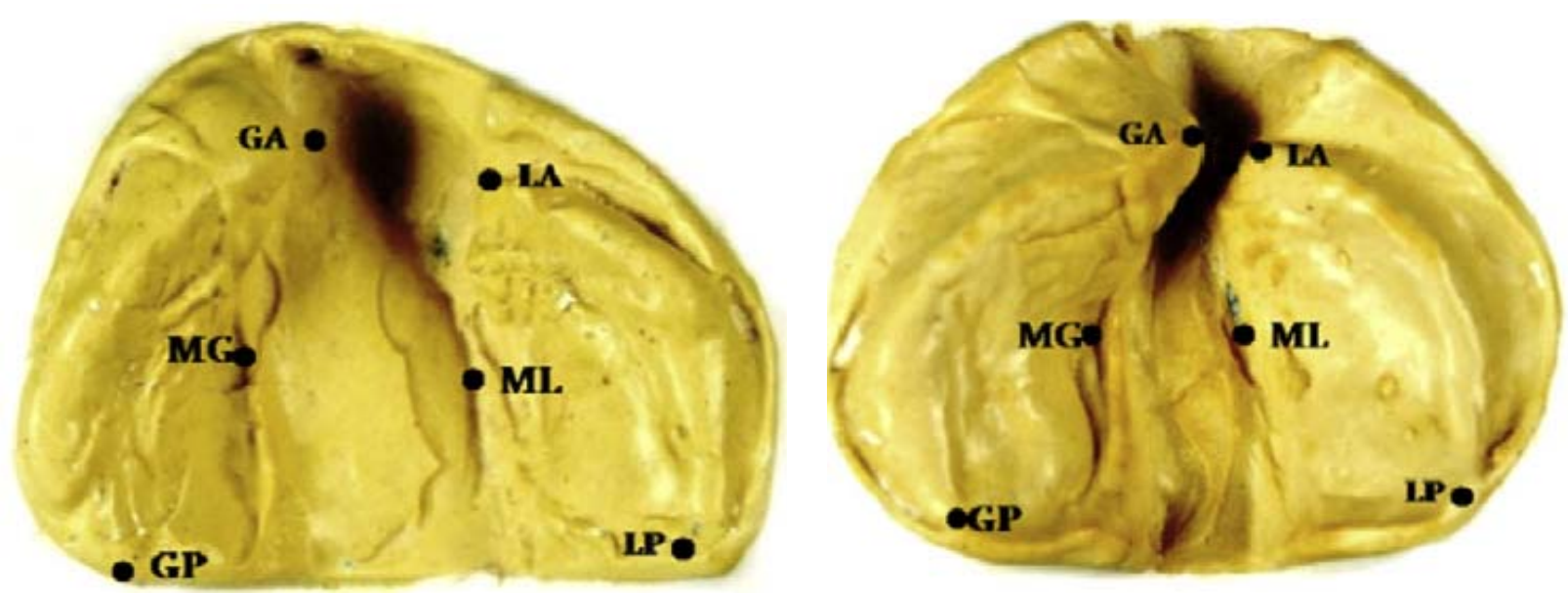

Figure 7. Showing pretreatment and post treatment model.

\section{Discussion}

Moulding plate therapy yielded a reduction of cleft width in the alveolar cleft area and in palatal area after a treatment period of 5 months. Follow up of the cases is continued even after the lip closure. The remarkable feature is that the physiologic growth of alveolar segments continues after lip closure in the desired direction without the molding plate. Physiologic forces from the closed lip with minimal scars continued to draw alveolar segments towards each other and the midline, leading to an almost complete elimination of the cleft alveolus gap. The noticeable feature is maintenance of the arch form without the collapse of alveolar segments. The proposition that favourable growth continues once it is directed physiologically is proved right by results achieved in these cases. This effect is most likely to result from the combined effect of redirection of growth of the alveolar 

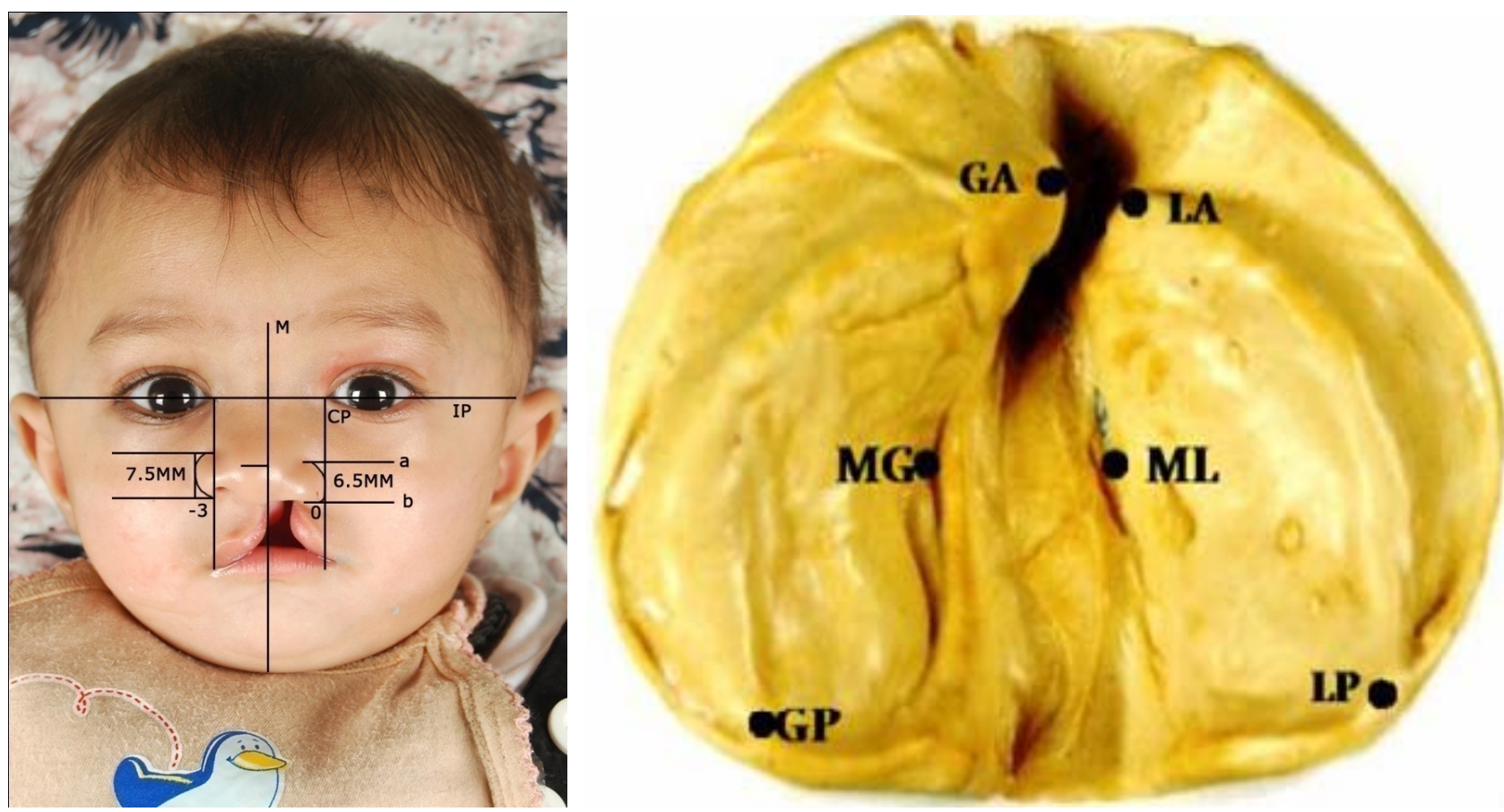

(a)
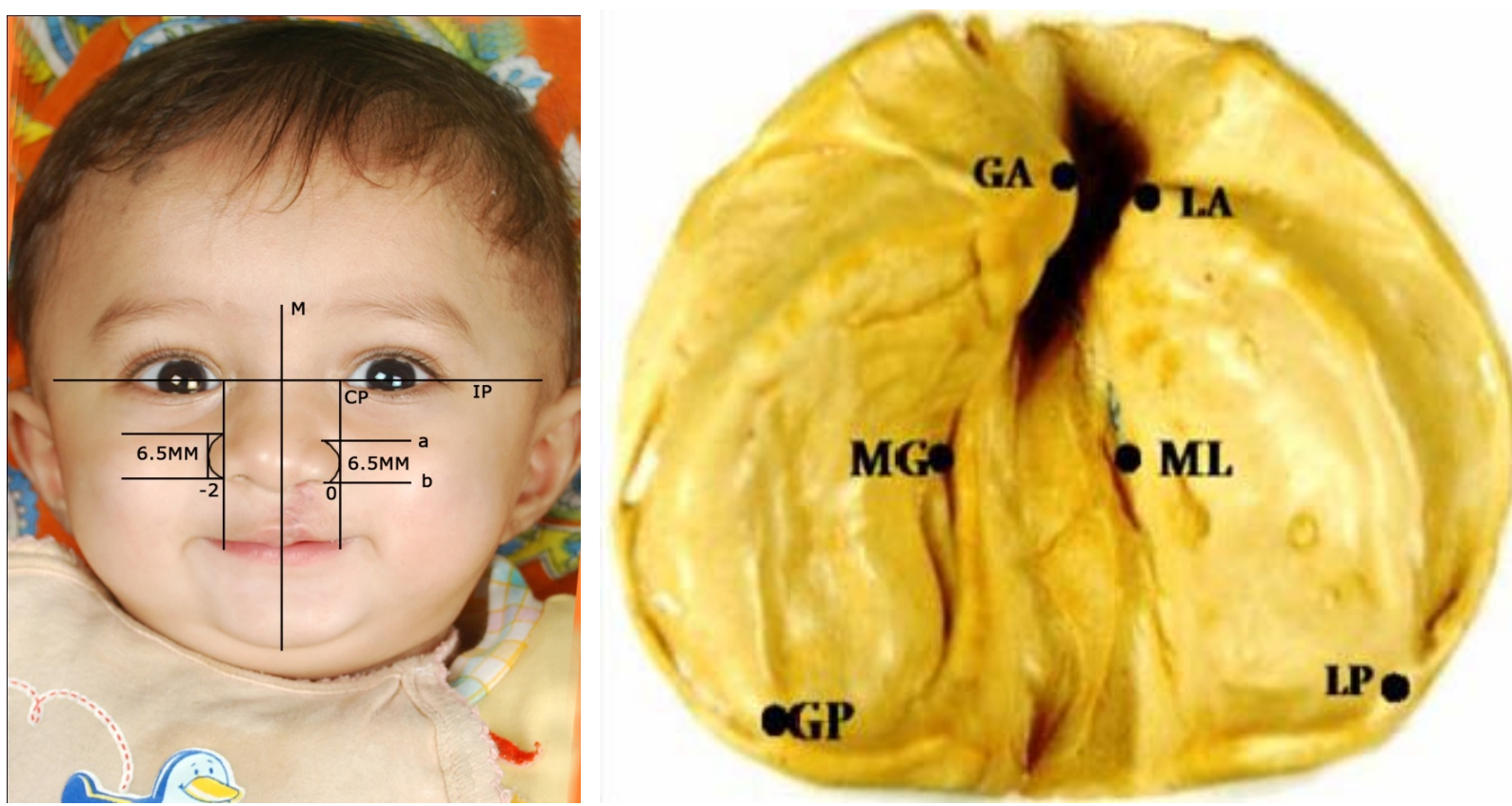

(b)

Figure 8. (a) Photograph and model before lip repair surgery; (b) Photograph and model after 1 month of surgery.

segments via the moulding plate, through active moulding by selective addition and removal of acrylic and prevention of tongue insertion into the cleft, leading to a separation of the cleft margins. Additional effects on the alveolar cleft were accomplished using adhesive tape tractions applied across the cleft lip as proposed by Grayson et al. (1999) [9].

Deng et al. (2005) reported cleft narrowing by $0.5 \mathrm{~mm}$ after a month's treatment, while Pai et al. (2005) [10] observed a reduction of $5.8 \mathrm{~mm}$ after 3 to 4 months of treatment. The reduction in cleft width is most likely to result from the combined effect of redirection of growth of the alveolar segments via the molding plate, through active moulding by selective addition and removal of acrylic and prevention of tongue insertion into the cleft, leading to a separation of the cleft margins. Additional 
effects on the alveolar cleft were accomplished using adhesive tape tractions applied across the cleft lip as proposed by Grayson et al. (1999) [2].

The reduction of cleft sided alar height did not proceed any further after 6 months postoperatively, which supported Liou et al. (2004) [11], who observed stable results even 1 year after surgery. Pre-surgical orthopaedics is a useful preliminary measure that should be carried out as it proves to be very cost effective and provides excellent aesthetic outcomes immediately after surgery.

\section{Conclusion}

PNAM should be considered soon after birth to promote a physiological pattern of function, which eventually sets the course for the functional patterns in later life. It effectively improves nasal symmetry in terms of nostril width, height and columella angle. Patients also benefit by a decrease in alveolar \& palatal gap width, thereby facilitating lip repair, followed by palatal closure with minimal surgical soft-tissue dissection. Hence, PNAM, when performed prior to primary lip repair, will give psychological reassurance to parents, enhance surgical outcome, reduce the need for soft-tissue revision surgeries later and also reduce the overall cost of treatment.

\section{REFERENCES}

[1] B. H. Grayson and C. B. Cutting, "Presurgical Nasoalveolar Orthopedic Molding in Primary Correction of the Nose, Lip, and Alveolus of Infants Born with Unilateral and Bilateral Clefts," The Cleft Palate-Craniofacial Journal, Vol. 38, No. 3, 2001, pp. 193-198.

doi:10.1597/1545-1569(2001)038<0193:PNOMIP $>2.0 . C$ $\underline{\mathrm{O} ; 2}$

[2] C. Lee, B. H. Grayson, W. Y. Lin and C. B. Cutting, "Long Term Study of Midface Growth in Unilateral Cleft Lip and Palate Patients Following Gingivoperiosteoplasty," American Cleft Palate Craniofacial Association, Chapel Hill, 1999.

[3] J. M. Converse, V. M. Hogan and F. E. Borton, "Secondary Deformities of Cleft Lip, Cleft Lip and Nose, and
Cleft Palate," In: J. M. Converse, Ed., Reconstructive Plastic Surgery, Saunders, Philadelphia, 1977.

[4] R. D. Bennum, C. Perandones, V. A. Sepliarsky, S. N. Chantiri, M. I. Aguirre and P. L. Dogliotti, "Nonsurgical Correction of Nasal Deformity in Unilateral Complete Cleft Lip: A Six-Year Follow-Up," Plastic \& Reconstructive Surgery, Vol. 104, No. 3, 1999, pp. 616-630. doi:10.1097/00006534-199909030-00002

[5] K. Matsuo, T. Hirose, T. Otagiri and N. Norose, "Repair of Cleft Lip with Nonsurgical Correction of Nasal Deformity in the Early Neonatal Period," Plastic \& Reconstructive Surgery, Vol. 83, No. 1, 1989, pp. 25-31. doi:10.1097/00006534-198901000-00006

[6] K. Matsuo and T. Hirose, "Preoperative Non-Surgical Over-Correction of Cleft Lip Nasal Deformity," British Journal of Plastic Surgery, Vol. 44, No. 1, 1991, pp. 5-11. doi:10.1016/0007-1226(91)90168-J

[7] A. Bajaj, K. S. Rao, S. M. Sharma and V. Shetty, "Modified Presurgical Nasoalveolar Molding in the Infants with Complete Unilateral Cleft Lip and Palate: A Stepwise Approach," Journal of Maxillofacial and Oral Surgery, Vol. 10, No. 3, 2011, pp. 275-280. doi:10.1007/s12663-011-0232-y

[8] M. Hotz, "Pre- and Early Postoperative Growth-Guidance in Cleft Lip and Palate Cases by Maxillary Orthopedics (an Alternative Procedure to Primary Bone Grafting)," The Cleft Palate Journal, Vol. 6, 1969, pp. 368-372.

[9] B. H. Grayson, P. E. Santiago, L. E. Brecht and C. B. Cutting, "Presurgical Nasoalveolar Molding in Infants with Cleft Lip and Palate," The Cleft Palate-Craniofacial Journal, Vol. 36, No. 6, 1999, pp. 486-498. doi:10.1597/1545-1569(1999)036<0486:PNMIIW>2.3.C $\underline{\mathrm{O} ; 2}$

[10] B. C. Pai, E. W. Ko, C. S. Huang and E. J. Liou, "Symmetry of the Nose after Presurgical Nasoalveolar Molding in Infants with Unilateral Cleft Lip and Palate: A Preliminary Study," The Cleft Palate-Craniofacial Journal, Vol. 42, No. 6, 2005, pp. 658-663. doi:10.1597/04-126.1

[11] D. Patel and R. Goyal, "Pre-Surgical Nasoalveolar Moulding in Patientwith Unilateral Cleft of Lip, Alveolus and Palate: Case Report," Journal of Plastic, Reconstructive \& Aesthetic Surgery, Vol. 65, No. 1, 2012, pp. 122-126. doi:10.1016/j.bjps.2011.06.011 